\title{
Effect of polar intermolecular interactions on the elastic constants of bent-core nematics and the origin of the twist-bend phase
}

\author{
M.A. Osipov ${ }^{1}$ and G. Pająk ${ }^{2, a}$ \\ 1 Department of Mathematics and Statistics, University of Strathclyde, Livingstone Tower, Richmond Street, Glasgow, Scotland, \\ UK \\ 2 M. Smoluchowski Institute of Physics, Department of Statistical Physics, Jagiellonian University, Łojasiewicza 11, 30-348 \\ Kraków, Poland
}

Received 10 January 2016 and Received in final form 26 February 2016

Published online: 26 April 2016

(C) The Author(s) 2016. This article is published with open access at Springerlink.com

\begin{abstract}
A molecular theory of both elastic constants and the flexoelectric coefficients of bent-core nematic liquid crystals has been developed taking into account dipole-dipole interactions as well as polar interactions determined by the bent molecular shape. It has been shown that if polar interactions are neglected, the elastic constants are increasing monotonically with the decreasing temperature. On the other hand, dipolar interactions between bent-core molecules may result in a dramatic increase of the bend flexocoefficient. As a result, the flexoelectric contribution to the bend elastic constant increases significantly, and the bend elastic constant appears to be very small throughout the nematic range and may vanish at a certain temperature. This temperature may then be identified as a temperature of the elastic instability of the bent-core nematic phase which induces a transition into the modulated phases with bend deformations like recently reported twist-bend phase. The temperature variation of the elastic constants is qualitatively similar to the typical experimental data for bent-core nematics.
\end{abstract}

\section{Introduction}

Nematic liquid crystals (LCs) are uniaxial anisotropic fluids composed of anisotropic molecules. Primary axes of such molecules are partially ordered along the local symmetry axis specified by the unit vector-director $\mathbf{n}$. Main properties of nematic LCs are relatively well understood, and at present the attention is focused into the so-called twist-bend nematic phase. Recently it has been found experimentally that some mesogenic dimers, in which rigid cores are connected by a flexible aliphatic chain, exhibit a transition into the novel low temperature nematic phase which is seemingly uniaxial [1-3]. This phase is characterized by macroscopic stripes with a period related to the cell thickness [1], and it has been found also in bent-core LCs $[4,5]$ and their hybrids [6]. It has been established by freeze-fracture transmission electron microscopy that the microscopic structure of the twist-bend phase is the oblique helicoid of the director with the extremely short pitch of approximately three molecular lengths [1-7]. Such a structure is consistent with strong twist and bend deformations. The properties of this phase have also been

\footnotetext{
${ }^{a}$ e-mail: grzegorz@th.if.uj.edu.pl
}

investigated by other techniques including small angle $\mathrm{x}-$ ray scattering and magnetic resonance $[8,9]$.

From the theoretical point of view different liquid crystal phases composed of bent-core molecules have been analysed using the Landau-de Gennes theory in $[10,11]$. The twist-bend phase has been comprehensively described by Dozov [12] who has assumed that in the nematic phase composed of bent-core molecules the bend elastic constant can approach zero and even turn negative at a certain temperature. In this case the homogeneous director distribution will become unstable because of the rapidly growing bend and twist deformations which are finally stabilized by the corresponding higher-order terms. Dozov has also showed that the modulated phase could be either a twist-bend (TB) or a splay-bend (SB) phase depending on the relationship between bend and splay elastic constants. The helical structure of the twist-bend phase has also been confirmed by simulations $[5,13,14]$. It should be noted that the key assumption about the decrease of the bend elastic constant has been indeed confirmed experimentally $[3,8,15]$. Clearly it is impossible to measure negative elastic constants, but very low positive values of $K_{33}$ have been observed directly above the transition into the TB phase $[3,8,15]$. 
It is impossible to develop a consistent theory of the TB phase without presenting a model which explains why the bend elastic constant decreases with the decreasing temperature and approaches zero at a certain temperature in the nematic phase. A reasonable model has recently been proposed by Selinger [16] who has taken into consideration that the Frank elastic constants are renormalized by the flexoelectric coefficients. In conventional nematics this effect is negligibly weak as the flexocoefficients are relatively small. However, in bent-core nematics and in nematics composed of mesogenic dimers the flexcoefficients may be significantly larger although the results obtained by different methods are controversial $[17,18]$. Selinger has shown that the flexoelectric coefficients may increase dramatically if the system is close to the transition into the virtual proper ferroelectric phase. Such a ferroelectric phase may never be observed as it is less stable then other phases, but the corresponding pretransitional effects should still be taken into account in the description of the flexoelectric effect. Close to the ferroelectric phase, a nematic liquid crystal possesses a very large dielectric susceptibility and hence any bend or splay deformations of the director should induce a large polarization. Recently the phenomenological model of the NTB phase, proposed by Selinger, has been used to calculate the compressibility of the NTB phase and to compare it with the experiment [19]. The model has also been generalised to account for the biaxial order of bent-core molecules [20]. Very recently a more general Landau-de Gennes theory has been developed which enables one to describe three different chiral nematic phases with phase transitions among them [21].

One notes that the theory [16] is phenomenological and contains a number of parameters which are not related to each other. Thus it describes the effect in principle but does not indicate how strong is the effect of flexcoefficients on the values of the elastic constants. An alternative theory has recently been developed by Ferrarini et al. [22] who have been able to obtain some numerical evidence of very low values of the elastic constants using the model of non-polar bent-core molecules, i.e. without taking flexoelectricity into account. Very recently it has been shown theoretically that the TB phase can be exhibited by an athermal system of hard bent rods [14]. One notes also that of the first molecular theories of uniaxial and biaxial nematic ordering in the system of bent rods has been presented by Teixeira et al. [23]. The relationship between the two approaches is briefly discussed in the last section. Finally, very recently another molecular theory of the locally polar bent-core nematic phase has been proposed by Vanakaras and Photinos [24] who have considered a nematic with a non-conical short pitch helical structure.

In this paper we use the same molecular model to calculate both the elastic constants and the flexoelectric coefficients of bent-core nematics using the molecularstatistical theory in the Parsons-Lee approximation. We present explicit expressions for the effective flexoelectric coefficients and demonstrate how they diverge on the approach to the transition into the virtual ferroelectric phase. Finally we calculate effective (renormalized) elas- tic constants as functions of temperature and show how the bend elastic constant $K_{33}$ may go to zero while $K_{11}$ and $K_{22}$ behave in a conventional way.

\section{Effect of flexoelectricity on the Frank elastic constants}

The distortion free energy of the polar nematic LC can be written in the following standard form:

$$
\begin{aligned}
F_{d}= & \frac{1}{2} K_{11} \mathbf{S}^{2}+\frac{1}{2} K_{22} \mathcal{T}^{2}+\frac{1}{2} K_{33} \mathbf{B}^{2}+\frac{1}{2} \mathbf{P} \cdot\left(\chi^{-1}\right) \cdot \mathbf{P} \\
& -e_{10}(\mathbf{P} \cdot \mathbf{S})-e_{30}(\mathbf{P} \cdot \mathbf{B}),
\end{aligned}
$$

where $\mathbf{S}=\mathbf{n}(\nabla \cdot \mathbf{n})$ is the splay deformation vector, $\mathbf{B}=(\mathbf{n} \times(\nabla \times \mathbf{n}))$ is the bend deformation vector and $\mathcal{T}=(\mathbf{n} \cdot(\nabla \times \mathbf{n}))$ is the twist deformation pseudoscalar. Here the first three terms represent the standard Frank elastic energy where $K_{11}, K_{22}, K_{33}$ are the "bare" elastic constants. The fourth term is the dielectric energy of the polar nematic, where $\chi_{i j}$ is the dielectric susceptibility tensor of the nematic phase which can be expressed as

$$
\chi_{i j}=\chi_{0} \delta_{i j}+\left(\chi_{\|}-\chi_{\perp}\right)\left(n_{i} n_{j}-\frac{1}{3} \delta_{i j}\right)
$$

and where $\chi_{\|}$is the longitudinal susceptibility of the nematic phase (along the director $\mathbf{n}), \chi_{\perp}$ is the transverse susceptibility (perpendicular to the director), while $\chi_{0}$ is the average susceptibility. Finally, the last two terms describe the flexoelectric effect according to Meyer [25] (see also recent review [26]) that is a coupling between the polarization $\mathbf{P}$ and the bend and splay deformation vectors. Here $e_{10}$ and $e_{30}$ are the splay and bend bare flexoelectric coefficients, respectively.

Minimization of the free energy (1) with respect to polarization $\mathbf{P}$ yields

$$
\mathbf{P}=\chi_{\|} e_{10} \mathbf{S}+\chi_{\perp} e_{30} \mathbf{B}
$$

where we have used eq. (2) and have taken into account that $(\mathbf{n} \cdot \mathbf{B})=0$. Substituting the polarization (3) back into the free energy (1) one obtains

$$
F_{d}=\frac{1}{2} \tilde{K}_{11} \mathbf{S}^{2}+\frac{1}{2} K_{22} \mathcal{T}^{2}+\frac{1}{2} \tilde{K}_{33} \mathbf{B}^{2},
$$

where $\tilde{K}_{11}$ and $\tilde{K}_{33}$ are the effective splay and bend elastic constants renormalized by the flexocoefficients:

$$
\tilde{K}_{11}=K_{11}-\frac{1}{2} \chi_{\|} e_{10}^{2} ; \quad \tilde{K}_{33}=K_{33}-\frac{1}{2} \chi_{\perp} e_{30}^{2} .
$$

One notes that the flexoelectric corrections to the elastic constants are negative and thus they diminish the values of the latter. On the other hand, for normal values of the flexoelectric coefficients the corrections are very small and may be neglected. This conclusion, however, is no longer valid if the system is close to the transition into the virtual ferroelectric phase which may actually not be observed. 
Indeed, above the transition into the ferroelectric phase the temperature variation of the dielectric susceptibility is described by the Curie-Weiss law, that is $\chi=\lambda /\left(T-T_{0}\right)$ where $T_{0}$ is the temperature of the transition into the virtual ferroelectric phase.

It is interesting to note also that in the anisotropic non-polar nematic phase both components of the dielectric susceptibility are described by the Curie-Weiss law, i.e. $\chi_{\|}=\lambda_{1} /\left(T-T_{\|}\right)$and $\chi_{\perp}=\lambda_{2} /\left(T-T_{\perp}\right)$, but the temperatures $T_{\|}$and $T_{\perp}$ are generally different. This means that the spontaneous polarization appears first in the direction parallel or perpendicular to the director depending on which of the temperatures $T_{\|}$and $T_{\perp}$ is higher. Then the system undergoes the transition into the ferroelectric phase at the temperature $T_{c}=\max \left(T_{\|}, T_{\perp}\right)$. This general assumption is actually confirmed by the molecular theory of the flexoelectric effect presented in the following section. In bent-core nematics the bend deformation dominates and it is reasonable to assume that $T_{\|}<T_{\perp}$. Then the effective bend elastic constant $\tilde{K}_{33}=K_{33}-\frac{\lambda_{2}}{2\left(T-T_{\perp}\right)} e_{30}^{2}$ decreases when temperature approaches $T_{\perp}$ and vanishes at some temperature $T *>T_{\perp}$.

In this paper we develop a molecular theory of both flexoelectricity and the elasticity of bent-core nematics using the same molecular model and investigate how the temperature variation of the elastic constants is affected by the bend molecular shape the permanent molecular dipole and other parameters of bent-core molecules.

\section{Molecular theory of elasticity of nematics without polar order}

A molecular theory of elasticity of nematic LCs can be developed using the density functional approach to the theory of nematic LCs (see, for example, [27-29]) and employing the gradient expansion of the orientational distribution function [30-32] (see also [33-35] and references therein on the earlier works). In this approach, the free energy of a liquid crystal, $F$, is a functional of the orientational distribution function $f$. The general structure of the functional $F[f]$ is not known, but the functional derivatives are known and are related to the direct correlation functions of the nematic phase.

The free energy of the distorted nematic liquid crystal can generally be written as a sum of two terms:

$$
F=W+H,
$$

where $W$ is the free energy of the system without intermolecular interactions. Without the external field the ideal gas free energy $W$ can be expressed as

$$
W=\rho k_{B} T \int f(\omega) \ln [f(\omega) \Lambda] \mathrm{d} \omega,
$$

where $\omega=(\mathbf{a}, \mathbf{b}), \rho$ is the number density of molecules, the unit vectors $\mathbf{a}$ and $\mathbf{b}$ are in the direction of the long and short molecular axis respectively, and $\Lambda$ is a constant.
The reduced free energy $H[f]$ is the generating functional for the direct correlation functions of the system:

$$
\begin{aligned}
& \frac{\delta(\beta H)}{\delta(\rho(\omega))}=-C_{1}(\omega)=-\ln \rho(\omega)+\beta \mu+\mathrm{const}, \\
& \frac{\delta^{2}(\beta H)}{\delta\left(\rho\left(\omega_{1}\right)\right) \delta\left(\rho\left(\omega_{2}\right)\right)}=-C_{2}\left(\omega_{1}, \omega_{2}\right),
\end{aligned}
$$

where $\rho(\omega)=\rho f(\omega), \beta=\frac{1}{k_{B} T}, \mu$ is the chemical potential and $C_{2}\left(\omega_{1}, \omega_{2}\right)$ is the direct pair correlation function of the liquid crystal.

The reduced free energy $H$ can be expressed in a simple analytical form by expanding around its value in the isotropic phase, that is by using the functional Taylor expansion of $H$ in terms of $\Delta f=f-f_{0}$ where $f_{0}=\frac{1}{8 \pi^{2}}$ is the one particle distribution function for biaxial molecules in the isotropic phase. Taking into account that $\frac{\partial F}{\partial f}=0$ one obtains [27-29]

$$
\begin{aligned}
F= & \rho k_{B} T \int f(\mathbf{x}) \ln [f(\mathbf{x}) \Lambda] \mathrm{d} \mathbf{x} \mathrm{d} \mathbf{r}-\frac{1}{2} k_{B} T \rho^{2} \\
& \times \int C_{2 I}\left(\mathbf{x}_{1}, \mathbf{r}_{12}, \mathbf{x}_{\mathbf{2}}\right) \Delta f\left(\mathbf{x}_{1}\right) \Delta f\left(\mathbf{x}_{2}\right) \mathrm{d} \mathbf{r}_{12} \mathrm{~d} \mathbf{x}_{1} \mathrm{~d} \mathbf{x}_{2} \mathrm{~d} \mathbf{r}_{1},
\end{aligned}
$$

where $\mathbf{x}=(\mathbf{a}, \mathbf{b}), \mathbf{r}_{12}$ is the intermolecular vector and $C_{2 I}\left(\mathbf{x}_{1}, \mathbf{r}_{12}, \mathbf{x}_{2}\right)$ is the direct pair correlation function of the isotropic phase.

The general form of the direct pair correlation function is not known but there exist a number of useful approximations which enable one to obtain some analytical results. In particular, in the Parsons-Lee approximation [36, 37] one assumes that the direct correlation function can be written in the scaled form, i.e. $C_{2 I}\left(\mathbf{x}_{1}, \mathbf{r}_{12}, \mathbf{x}_{2}\right)=$ $C_{2}\left(r_{12} / \sigma_{12}\right)$ where $\sigma_{12}=\sigma_{12}\left(\mathbf{x}_{\mathbf{1}}, \mathbf{u}_{12}, \mathbf{x}_{\mathbf{2}}\right)$ is the minimum distance of approach for two rigid molecules "1" and " 2 " which depends on their relative orientation, and where the unit vector $\mathbf{u}_{12}=\mathbf{r}_{12} / r_{12}$. This approximation, however, makes sense only for molecules of convex shape when the function $\sigma_{12}$ is well defined. Simple interpolation (between three orientations, see for instance [38]) of $\sigma_{12}$ for two uniaxial hard spherocylinders, of diameter $d$ and with $L$ being its total length, is the following:

$$
\sigma_{12}=d+\frac{L-d}{2}\left(\left(\mathbf{a}_{1} \cdot \mathbf{u}_{12}\right)^{2}+\left(\mathbf{a}_{2} \cdot \mathbf{u}_{12}\right)^{2}\right) .
$$

In the case of bent-core molecules the Parsons-Lee approximation can be modified in the following way. Let us consider a simple model of a bent-core molecules (see fig. 1) which is composed of the two uniaxial arms rigidly joined together at a bend angle $\alpha$. Now let us assume that the pair correlation function for bent-core molecules $i$ and $j$ can be written as a sum of the correlation functions between different arms of the two molecules expressed in the Parsons-Lee approximation:

$$
C_{2 I}\left(\mathbf{x}_{\mathbf{i}}, \mathbf{r}_{i j}, \mathbf{x}_{\mathbf{j}}\right) \approx \sum_{\nu, \mu} C_{2}\left(\frac{r_{\nu \mu}}{\sigma_{\nu \mu}}\right)
$$




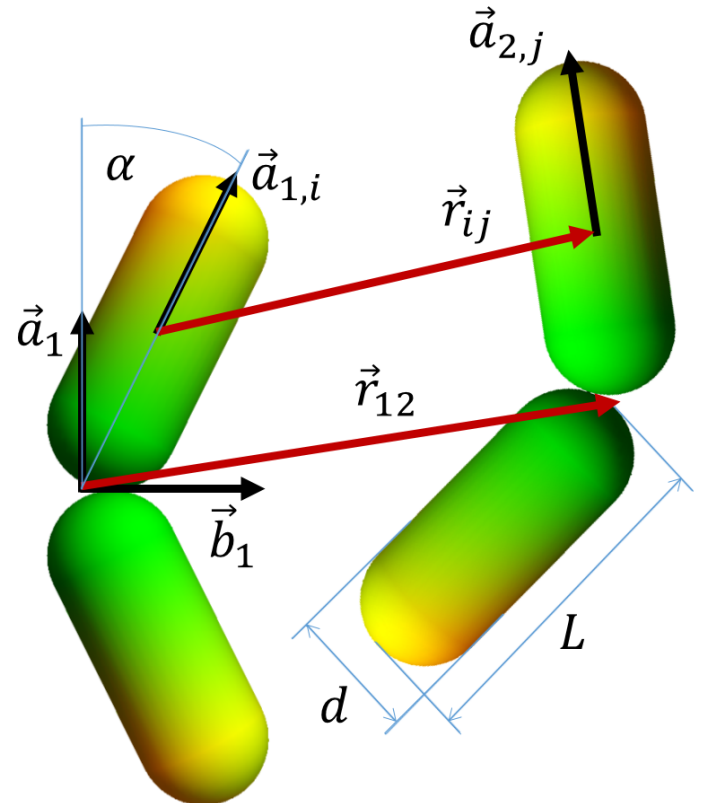

Fig. 1. (Color online) The model of hard bent-core molecules composed of two uniaxial spherocylinders of width $d$, where $L$ is its length. The opening angle of a molecule is equal to $\pi-2 \alpha$ and the distance between molecules 1 and 2 is given by the relation $\mathbf{r}_{12}=\mathbf{r}_{i j}+L\left(\mathbf{a}_{1, i}-\mathbf{a}_{2, j}\right) / 2$, where indices $i$ and $j$ correspond to the vectors along the arms located in the half-length of each spherocylinder.

where $\sigma_{\nu \mu}=\sigma_{\nu \mu}\left(\mathbf{a}_{i, \nu}, \mathbf{u}_{\nu \mu}, \mathbf{a}_{j, \mu}\right)$. Here $\mathbf{a}_{i, \nu}$ is the unit vector along the primary axis of the arm $\nu$ of the molecule $i, \mathbf{a}_{j, \mu}$ is the primary axis of the arm $\mu$ of the molecule $j$, $\mathbf{r}_{\nu \mu}$ is the vector between the centre of the arm $\nu$ of the molecule $i$ and the centre of the arm $\mu$ of the molecule $j$ and $\mathbf{u}_{\nu \mu}=\mathbf{r}_{\nu \mu} / r_{\nu \mu}$.

One notes that the approximation (12) is rather crude as it does not account for the correlations between three different arms, that is for the configurations, for example, when an arm is in contact with both arms of the neighboring molecule. On the other hand, the relative statistical weight of such configurations is sufficiently small and may be neglected in the first approximation. Introducing the new variables $\mathbf{r}_{\nu \mu}^{\prime}=\mathbf{r}_{\nu \mu} / \sigma_{\nu \mu}$ the second term in eq. (10) can be written in the form

$$
\begin{aligned}
H= & -\frac{1}{2} k_{B} T \rho^{2} \int \mathrm{d} \mathbf{r}_{1} \sum_{\nu, \mu} \int_{1}^{\infty} C_{2}\left(r_{\nu \mu}^{\prime}\right)\left(r_{\nu \mu}^{\prime}\right)^{2} \\
& \times\left(\int \Delta f\left(\mathbf{x}_{1}\right) \Delta f\left(\mathbf{x}_{2}\right) \sigma_{\nu \mu}^{3} \mathrm{~d} \mathbf{x}_{1} \mathrm{~d} \mathbf{x}_{2}\right) \mathrm{d} r_{\nu \mu}^{\prime} \mathrm{d} \mathbf{u}_{\nu \mu},
\end{aligned}
$$

where only the product $\Delta f\left(\mathrm{x}_{1}\right) \Delta f\left(\mathrm{x}_{2}\right) \sigma_{\nu \mu}^{3}$ depends on the orientational variables $\mathbf{x}_{1}, \mathbf{x}_{2}$. Equation (13) describes the reduced free energy of an inhomogeneous nematic phase. The distortion free energy can be separated from the free energy of the homogeneous state by employing the gradient expansion of the orientational distribution function $f\left(\mathbf{x}, \mathbf{n}\left(\mathbf{r}_{2}\right)\right)$ around the point $\mathbf{r}_{1}$ :

$$
\begin{aligned}
f\left(\mathbf{x}, \mathbf{n}\left(\mathbf{r}_{2}\right)\right)= & f\left(\mathbf{x}, \mathbf{n}\left(\mathbf{r}_{1}\right)\right)+\left(\mathbf{r}_{12} \cdot \nabla\right) f\left((\mathbf{a} \cdot \mathbf{n})^{2},(\mathbf{b} \cdot \mathbf{n})^{2}\right) \\
& +\frac{1}{2}\left(\mathbf{r}_{12} \cdot \nabla\right)^{2} f\left((\mathbf{a} \cdot \mathbf{n})^{2},(\mathbf{b} \cdot \mathbf{n})^{2}\right)+\ldots
\end{aligned}
$$

Neglecting the terms linear in gradients (which only contribute to the free energy of chiral nematics), eq. (14) can be rewritten as

$$
\begin{aligned}
f\left(\mathbf{x}, \mathbf{n}\left(\mathbf{r}_{2}\right)\right)-f\left(\mathbf{x}, \mathbf{n}\left(\mathbf{r}_{1}\right)\right) \approx & \frac{1}{2} \frac{\partial f}{\partial \mathbf{n}} \cdot\left(\mathbf{r}_{12} \cdot \nabla\right)^{2} \mathbf{n} \\
& +\frac{1}{2} \frac{\partial^{2} f}{\partial \mathbf{n}^{2}}:\left(\left(\mathbf{r}_{12} \cdot \nabla\right) \mathbf{n}\right)^{2}
\end{aligned}
$$

In this paper we will limit ourselves to the terms quadratic in $\mathbf{a}_{\nu}, \mathbf{a}_{\mu}$ and therefore will only employ the quadrupolar orientational order parameters $S=\left\langle P_{2}(\mathbf{a} \cdot \mathbf{n})\right\rangle$ and $D=\left\langle P_{2}(\mathbf{b} \cdot \mathbf{n})-P_{2}(\mathbf{c} \cdot \mathbf{n})\right\rangle$. In eq. (15) $\mathbf{r}_{12}=$ $\mathbf{r}_{\nu \mu} \pm(L / 2)\left(\mathbf{a}_{\nu}-\mathbf{a}_{\mu}\right)$. However, the second term in this equation can be neglected in the first approximation as it results in additional terms in the free energy which are higher order in $\mathbf{a}_{\nu}, \mathbf{a}_{\mu}$. Substituting $\mathbf{r}_{12}=\mathbf{r}_{\nu \mu} \sigma_{\nu \mu}$ and $\mathbf{u}_{12}=\mathbf{u}_{\nu \mu}$ into eqs. (15), (13), one obtains the following expression for the free energy of the inhomogeneous nematic:

$$
F=F_{0}+\int F_{d}(\mathbf{r}) \mathrm{d} \mathbf{r},
$$

where $F_{0}$ is the free energy of the homogeneous nematic and the distortion free energy $F_{d}$ is expressed as

$$
\begin{aligned}
F_{d}(\mathbf{r})= & -\frac{1}{2} k_{B} T \rho^{2} \lambda \sum_{\nu, \mu} \int \sigma_{\nu \mu}^{5} f\left(\mathbf{x}_{1}\right)\left(\frac{\partial f}{\partial \mathbf{n}} \cdot\left(\mathbf{u}_{\nu \mu} \cdot \nabla\right)^{2} \mathbf{n}\right. \\
& \left.+\frac{\partial^{2} f}{\partial \mathbf{n}^{2}}:\left(\left(\mathbf{u}_{\nu \mu} \cdot \nabla\right) \mathbf{n}\right)^{2}\right) \mathrm{d} \mathbf{x}_{1} \mathrm{~d} \mathbf{x}_{2} \mathrm{~d} \mathbf{u}_{\nu \mu}
\end{aligned}
$$

where

$$
\lambda=-\int_{1}^{\infty} C_{2}(r) r^{5} \mathrm{~d} r .
$$

The derivatives in eq. (17) with respect to the director $\mathbf{n}$ can be taken out of the integral over $\mathbf{x}_{2}$ :

$$
\begin{aligned}
F_{d}(\mathbf{r})= & \frac{1}{2} k_{B} T \rho^{2} \lambda \sum_{\nu, \mu} \int \mathrm{d} \mathbf{x}_{1} f\left(\mathbf{x}_{1}\right) \\
& \times\left(\frac{\partial}{\partial \mathbf{n}} \int f\left(\mathbf{x}_{2}\right) \sigma_{\nu \mu}^{5} \cdot\left(\mathbf{u}_{\nu \mu} \cdot \nabla\right)^{2} \mathbf{n} \mathrm{d} \mathbf{x}_{2} \mathrm{~d} \mathbf{u}_{\nu \mu}\right. \\
& \left.+\frac{\partial^{2}}{\partial \mathbf{n}^{2}}: \int f\left(\mathbf{x}_{2}\right) \sigma_{\nu \mu}^{5}\left(\left(\mathbf{u}_{\nu \mu} \cdot \nabla\right) \mathbf{n}\right)^{2}\right) \mathrm{d} \mathbf{x}_{2} \mathrm{~d} \mathbf{u}_{\nu \mu} .
\end{aligned}
$$

In eq. (18) the function $\sigma_{\nu \mu}$ depends only on $\left(\mathbf{a}_{\nu} \cdot \mathbf{u}_{\nu \mu}\right)$ and $\left(\mathbf{a}_{\mu} \cdot \mathbf{u}_{\nu \mu}\right)$ and thus it can be expanded in Legendre polynomials $P_{n}\left(\mathbf{a}_{\nu} \cdot \mathbf{u}_{\nu \mu}\right)$ and $P_{n}\left(\mathbf{a}_{\mu} \cdot \mathbf{u}_{\nu \mu}\right)$. The lowestorder term which contributes to the elastic constants is 
proportional to the product $P_{2}\left(\mathbf{a}_{\nu} \cdot \mathbf{u}_{\nu \mu}\right) P_{2}\left(\mathbf{a}_{\mu} \cdot \mathbf{u}_{\nu \mu}\right)$ as the relevant contributions must depend both on $\mathbf{a}_{\nu}$ and $\mathbf{a}_{\mu}$. The corresponding term in the expansion of $\sigma_{\nu \mu}^{5}$ comes with the following coefficient:

$$
\frac{20}{9} d^{3}(L-d)^{2} P_{2}\left(\mathbf{a}_{\nu} \cdot \mathbf{u}_{\nu \mu}\right) P_{2}\left(\mathbf{a}_{\mu} \cdot \mathbf{u}_{\nu \mu}\right) .
$$

Substituting eq. (19) into (18) one obtains

$$
\begin{aligned}
F_{d}(\mathbf{r})= & k_{B} T \rho^{2} \lambda \tilde{S}^{2} \kappa P_{2}(\mathbf{u} \cdot \mathbf{n}) \int\left(\frac{\partial P_{2}(\mathbf{u} \cdot \mathbf{n})}{\partial \mathbf{n}} \cdot(\mathbf{u} \cdot \nabla)^{2} \mathbf{n}\right. \\
& \left.+\frac{\partial^{2} P_{2}(\mathbf{u} \cdot \mathbf{n})}{\partial \mathbf{n}^{2}}:((\mathbf{u} \cdot \nabla) \mathbf{n})^{2}\right) \mathrm{d} \mathbf{u} \\
= & 3 k_{B} T \rho^{2} \lambda \tilde{S}^{2} \kappa P_{2}(\mathbf{u} \cdot \mathbf{n}) \int\left((\mathbf{u} \cdot \mathbf{n})\left(\mathbf{u} \cdot(\mathbf{u} \cdot \nabla)^{2} \mathbf{n}\right)\right. \\
& \left.+(\mathbf{u} \cdot(\mathbf{u} \cdot \nabla) \mathbf{n})^{2}\right) \mathrm{d} \mathbf{u},
\end{aligned}
$$

where

$$
\kappa=\frac{20}{9} d^{3}(L-d)^{2}
$$

and where we have taken into consideration that in the first approximation $\mathbf{u}_{\nu \mu}=\mathbf{u}$. Here the order parameter $\tilde{S}$ is expressed as

$$
\begin{gathered}
\tilde{S}=\left\langle P_{2}\left(\mathbf{a}_{i} \cdot \mathbf{n}\right)\right\rangle=\frac{3}{2}\left\langle(\mathbf{a} \cdot \mathbf{n})^{2}\right\rangle \cos ^{2} \alpha+\frac{3}{2}\left\langle(\mathbf{b} \cdot \mathbf{n})^{2}\right\rangle \sin ^{2} \alpha \\
-\frac{1}{2}=S \cos ^{2} \alpha+\frac{1}{2}(1-S+D) \sin ^{2} \alpha
\end{gathered}
$$

where $\mathbf{a}_{i}= \pm \mathbf{a} \cos \alpha+\mathbf{b} \sin \alpha$ is the unit vector in the direction of the long axis of an arm $i$ and where $\mathbf{a}$ and $\mathbf{b}$ is the long and short axis of the whole bent-core molecule, respectively. Here $S=\left\langle P_{2}(\mathbf{a} \cdot \mathbf{n})\right\rangle$ and $D=\left\langle(\mathbf{b} \cdot \mathbf{n})^{2}\right\rangle-$ $\left\langle(\mathbf{c} \cdot \mathbf{n})^{2}\right\rangle$ are the two orientational order parameters of the biaxial bent-core molecule in the uniaxial nematic phase and $\mathbf{c}$ is the second short molecular axis.

One notes that the first term in eq. (20) contains second derivatives of the director while the distortion free energy depends only of the square of the first derivatives. This is related to the fact that eq. (20) contains both bulk and surface elasticity of the nematic. The surface and bulk contributions can be separated by taking the integral of the first term in eq. (20) over $\mathbf{r}_{1}$ by parts in the expression for the total distortion energy of the whole sample $F_{d}=\int F_{d}(\mathbf{r}) \mathrm{d} \mathbf{r}$ :

$$
\begin{aligned}
& \int\left[3 k_{B} T \rho^{2} \lambda \tilde{S}^{2} \kappa P_{2}(\mathbf{u} \cdot \mathbf{n})\right. \\
& \left.\times \int(\mathbf{u} \cdot \mathbf{n})\left(\mathbf{u} \cdot(\mathbf{u} \cdot \nabla)^{2} \mathbf{n}\right) \mathrm{d} \mathbf{u}\right] \mathrm{d} \mathbf{r}= \\
& -\int\left[3 k_{B} T \rho^{2} \lambda \tilde{S}^{2} \kappa \int\left(P_{2}(\mathbf{u} \cdot \mathbf{n})+(\mathbf{u} \cdot \mathbf{n})^{2}\right)\right. \\
& \times((\mathbf{u} \cdot(\mathbf{u} \cdot \nabla) \mathbf{n}))] \mathrm{d} \mathbf{r}+(\text { surface terms }) .
\end{aligned}
$$

The first term in eq. (23) depends on the square of the first derivatives of the director and describes a contribution to the bulk distortion free energy of the nematic phase.
Now eqs. (20) and (23) can be used to obtain explicit expressions for the elastic constants. As shown in [30], in the case of a slow variation of the director in the $(x, z)$ plane the only non-zero gradients for the splay, twist and bend deformations are $\nabla_{x} n_{x}=q, \nabla_{y} n_{x}=q$ and $\nabla_{z} n_{x}=q$, respectively. Substituting these expressions in to eq. (23) one obtains explicit expressions for the elastic constants:

$$
\begin{aligned}
K_{11}= & \frac{1}{4} k_{B} T \rho^{2} \lambda \tilde{S}^{2} \kappa \int \mathrm{d} \mathbf{u} \\
& \times\left(3 P_{2}\left(u_{z}\right) u_{x}^{4}-\frac{9}{2} u_{z}^{2} u_{x}^{4}+\frac{1}{2} u_{x}^{4}\right) ; \\
K_{22}= & \frac{1}{4} k_{B} T \rho^{2} \lambda \tilde{S}^{2} \kappa \int \mathrm{d} \mathbf{u} \\
& \times\left(3 P_{2}\left(u_{z}\right) u_{x}^{2} u_{y}^{2}-\frac{9}{2} u_{z}^{2} u_{x}^{2} u_{y}^{2}+\frac{1}{2} u_{x}^{2} u_{y}^{2}\right) ; \\
K_{33}= & \frac{1}{4} k_{B} T \rho^{2} \lambda \tilde{S}^{2} \kappa \int \mathrm{d} \mathbf{u} \\
& \times\left(2 P_{2}\left(u_{z}\right) u_{x}^{2} u_{z}^{2}-\frac{9}{2} u_{z}^{4} u_{x}^{2}+\frac{1}{2} u_{x}^{2} u_{z}^{2}\right) .
\end{aligned}
$$

Taking integrals over $\mathbf{u}$ one obtains final expressions for the elastic constants in the relatively simple form:

$$
K_{11}=-k_{B} T \frac{\pi}{5} \rho^{2} \lambda \kappa \tilde{S}^{2}
$$

and

$$
K_{22}=K_{33}=-k_{B} T \frac{\pi}{15} \rho^{2} \lambda \kappa \tilde{S}^{2} .
$$

The corresponding figures of these elastic constants are presented in fig. 2, where we have introduced the parameter $\rho^{*}=\rho d^{2} L$ which allows to draw all curves in reduced (depending only on parameter $\alpha$ ) variables $K_{i i}^{\text {red }}=\frac{d}{-\lambda t J\left(\rho^{*}\right)^{2}} K_{i i}$ as a function of dimensionless temperature $t=\frac{k_{B} T}{J}$ for Maier-Saupe dependence of $S(t)$ and $\tilde{S}(t)$. In these figures the expected result is with decreasing opening angle of bent-core particle, that is larger value of angle $\alpha$, the absolute values of elastic constants are also decreasing, consequently providing maximum for rodlike shapes. In this approximation $K_{22}=K_{33}$, and this is related to the fact that we have taken into account only terms quadratic in a. It is well known that the difference between $K_{22}$ and $K_{33}$ is determined by the higher orientational order parameters including, in particular, $\left\langle P_{4}(\mathbf{a} \cdot \mathbf{n})\right\rangle$. However, equations present the so called "bare" elastic constants which have not been renormalized by the flexoelectric effect. Our final expressions for the elastic constants, presented in the following section, distinguish between $K_{22}$ and $K_{33}$.

\section{Theory of the flexoelectric effect in bent-core nematics}

In the distorted liquid crystal the orientational distribution function is modified, and in the case of small orientational deformation it contains a small correction $h(\mathbf{a}, \mathbf{b}, \mathbf{n})$ 

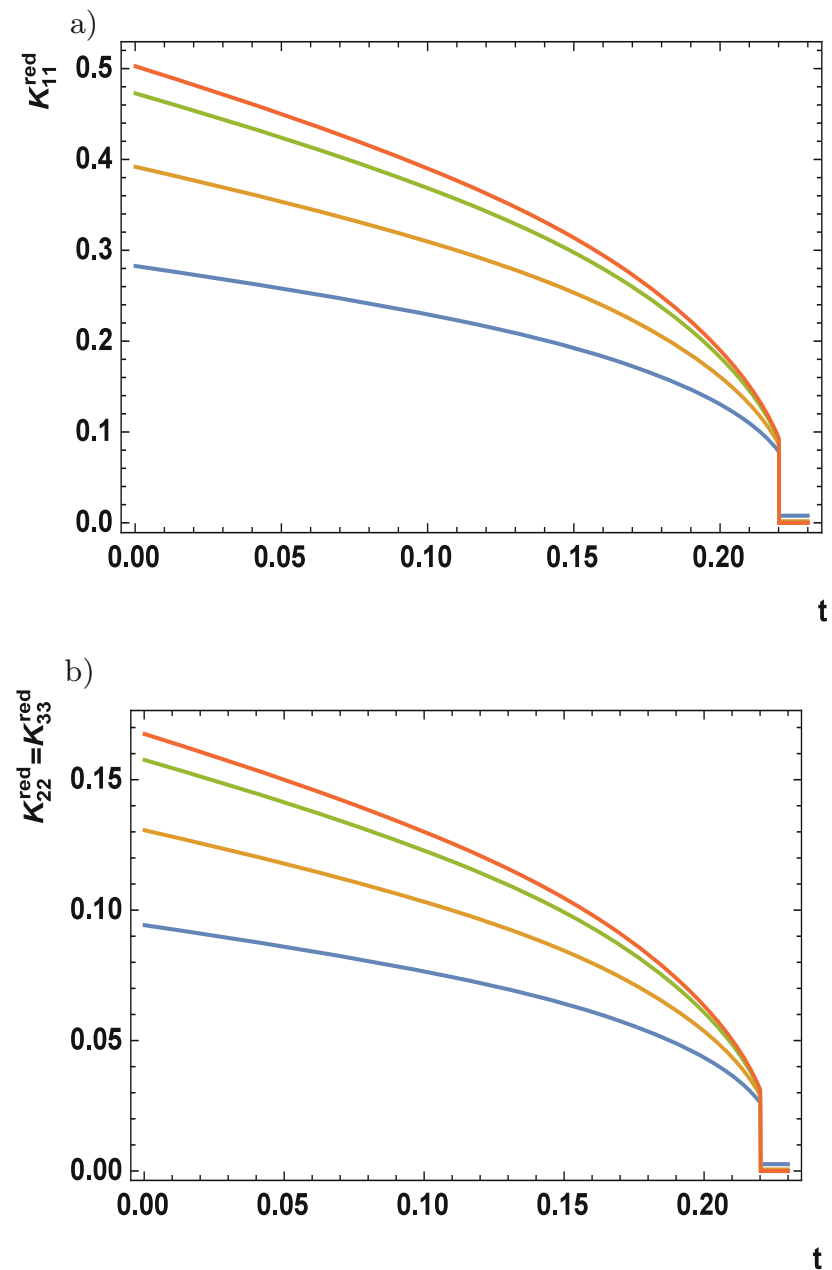

Fig. 2. (Color online) Reduced splay (a) twist and bend (b) elastic constants $K_{i i}^{\mathrm{red}}=\frac{d}{-\lambda t J\left(\rho^{*}\right)^{2}} K_{i i}$, where $K_{i i}$ are given by eq. (27) and eq. (28) for $L=2.5 d$ and for four opening angles from the very top: $180^{\circ}$ (red), $160^{\circ}$ (green), $140^{\circ}$ (orange) and $120^{\circ}$ (blue), which correspond to parameter $\alpha$ equal to $0, \frac{\pi}{18}$, $\frac{\pi}{9}$ and $\frac{\pi}{6}$, respectively.

which is generally polar:

$$
f(\mathbf{a}, \mathbf{b}, \mathbf{n})=f_{0}(\mathbf{a}, \mathbf{b}, \mathbf{n})(1+h(\mathbf{a}, \mathbf{b}, \mathbf{n})),
$$

where $f_{0}(\mathbf{a}, \mathbf{b}, \mathbf{n})$ is the orientational distribution function of the undistorted nematic phase and the small correction $h=h_{f}+h_{p}$ is a sum of two terms. The function $h_{f} \sim \nabla_{\alpha} n_{\beta}$ is proportional to the gradients of the director while the function $h_{p} \sim\langle\mathbf{b}\rangle$ is proportional to the polar order parameter $\langle\mathbf{b}\rangle$ which determines the polarization due to the polar ordering of short molecular axes. One notes that another polar order parameter $\langle\mathbf{a}\rangle=0$ because $\mathbf{a}$ is always equivalent to $-\mathbf{a}$ due to the $C_{2 v}$ symmetry of a bent-core molecule. Then the average molecular dipole in the distorted nematic phase can be expressed as:

$$
\langle\mathbf{d}\rangle=\int \mathbf{d} f_{0}(\mathbf{a}, \mathbf{b}, \mathbf{n}) h(\mathbf{a}, \mathbf{b}, \mathbf{n}) \mathrm{d} \mathbf{b} \mathrm{d} \mathbf{a},
$$

where the molecular dipole

$$
\mathbf{d}=\mu_{\perp} \mathbf{b} .
$$

Here the polar correction to the orientational distribution function $h(\mathbf{a}, \mathbf{b}, \mathbf{n})$ should be determined using a corresponding molecular-statistical theory.

The one-particle distribution function of the distorted liquid crystal can be determined by minimization of the free energy with respect to $f(\omega)$ taking into account the normalization condition. One obtains

$$
\begin{aligned}
\delta\left(F+\lambda \int \rho(\omega) \mathrm{d} \omega\right) \delta \rho(\omega)= & -k_{B} T C_{1}(\omega)+k_{B} T \ln f(\omega) \\
& +\Lambda+\lambda=0
\end{aligned}
$$

where the lagrange multiplier $\lambda$ is determined from the normalization condition, $f(\omega)$ is the one-particle distribution of the distorted nematic, given by eq. (29) and $C_{1}(\omega)$ is the one-particle correlation function. Let us now perform the functional Taylor expansion of the function $C_{1}(\rho(\omega))$ in the right hand side of eq. (32) about the local distribution $f_{0}(\omega)$ :

$$
\begin{aligned}
C_{1}(\rho)= & C_{1}\left(\rho_{0}(\omega)\right)+\rho \int C_{2}\left(\rho_{0}\left(\omega_{1}\right), \rho_{0}\left(\omega_{2}\right)\right) \\
& \times\left(f\left(\omega_{2}\right)-f_{0}\left(\omega_{2}\right)\right) \mathrm{d} \omega_{2}+\ldots,
\end{aligned}
$$

where $C_{2}\left(\rho_{0}\left(\omega_{1}\right), \rho_{0}\left(\omega_{2}\right)\right)$ is the pair direct correlation function. One notes that he higher-order terms in the expansion (which are determined by higher-order direct correlation functions) do not contribute to the flexoelectric effect because they are proportional to the higher powers of $\left(f\left(\omega_{2}\right)-f_{0}\left(\omega_{2}\right)\right)$ and hence to the higher powers of the orientational deformation.

The difference $\left(f\left(\omega_{2}\right)-f_{0}\left(\omega_{2}\right)\right)$ in the second term in the right hand side of eq. (33) can be expressed as $\left(f(\omega)-f_{0}(\omega)\right)=f_{0}\left((\mathbf{a} \cdot \mathbf{n})^{2},(\mathbf{b} \cdot \mathbf{n})^{2}\right) h(\omega)$ by using eq. (29). Performing the gradient expansion of the function $f_{0}\left((\mathbf{a} \cdot \mathbf{n})^{2},(\mathbf{b} \cdot \mathbf{n})^{2}\right)$ to the first order, one finally obtains

$$
\begin{aligned}
\left(f(\omega)-f_{0}(\omega)\right)= & \left(\mathbf{r}_{12} \cdot \nabla\right) f_{0}\left((\mathbf{a} \cdot \mathbf{n})^{2},(\mathbf{b} \cdot \mathbf{n})^{2}\right) \\
& +f_{0}\left((\mathbf{a} \cdot \mathbf{n})^{2},(\mathbf{b} \cdot \mathbf{n})^{2}\right) h(\omega) .
\end{aligned}
$$

Substituting eq. (34) into eq. (33) and then into the general eq. (32) and expanding $\ln f$ in eq. (32) to the first order of the small function $h$ one obtains the following integral equation for the correction $h(\omega)$ :

$$
\begin{aligned}
h\left(\mathbf{x}_{1}\right)= & \rho \int C_{2}\left(\mathbf{x}_{1}, \mathbf{x}_{2}, \mathbf{r}_{12}\right)\left(\mathbf{r}_{12} \cdot \nabla\right) \\
& \times f_{0}\left((\mathbf{a} \cdot \mathbf{n})^{2},(\mathbf{b} \cdot \mathbf{n})^{2}\right) \mathrm{d} \mathbf{x}_{2} \mathrm{~d} \mathbf{r}_{12} \\
& +\rho \int C_{2}\left(\mathbf{x}_{1}, \mathbf{x}_{2}, \mathbf{r}_{12}\right) f_{0}\left((\mathbf{a} \cdot \mathbf{n})^{2},(\mathbf{b} \cdot \mathbf{n})^{2}\right) \\
& \times h\left(\mathbf{x}_{2}\right) \mathrm{d} \mathbf{x}_{2} \mathrm{~d} \mathbf{r}_{12},
\end{aligned}
$$

where $\mathbf{x}=(\mathbf{a}, \mathbf{b})$.

In the general case the correction $h(\mathbf{a}, \mathbf{b})$ is a sum of the "symmetric" and the "antisymmetric" parts:

$$
h(\mathbf{a}, \mathbf{b})=h_{s}(\mathbf{a}, \mathbf{b})+h_{a}(\mathbf{a}, \mathbf{b})
$$

where the symmetric part $h_{s}(\mathbf{a}, \mathbf{b})$ is non-polar, i.e. it is invariant under the transformation $\mathbf{b} \rightarrow-\mathbf{b}$ while the 
antisymmetric part $h_{a}(\mathbf{a}, \mathbf{b})$ changes sign under such a transformation. The flexoelectric effect is determined only by the antisymmetric function $h_{a}(\mathbf{a}, \mathbf{b})$ which is sensitive to the direction of the molecular dipole. Equation (35) can now be split into two independent equations for the functions $h_{s}(\mathbf{a}, \mathbf{b})$ and $h_{a}(\mathbf{a}, \mathbf{b})$. Because the last term in eq. (35) vanishes when $h(\mathbf{a}, \mathbf{b})=h_{a}(\mathbf{a}, \mathbf{b})$ provided the direct correlation function $C_{2}\left(\mathbf{x}_{1}, \mathbf{x}_{2}, \mathbf{r}_{12}\right)$ is non-polar. On the other hand, in the system of polar molecules the direct correlation function should contain a polar contribution which is determined both by polar molecular shape and dipolar intermolecular interactions. Thus the last term contributes to the equation for the polar correction to the distribution function.

In this section we assume that the direct correlation function can be expressed as

$$
\begin{aligned}
C_{2 I}\left(\mathbf{x}_{1}, \mathbf{r}_{12}, \mathbf{x}_{2}\right) \approx & \sum_{\nu, \mu} C_{2}\left(\frac{r_{\nu \mu}}{\sigma_{\nu \mu}}\right) \\
& -\frac{1}{k_{B} T} \Theta\left(r_{12}-d\right) U_{d d}\left(\mathbf{b}_{1}, \mathbf{b}_{2}, \mathbf{r}_{12}\right),
\end{aligned}
$$

where the first term is a sum of the correlation functions between different arms of the two molecules expressed in the Parsons-Lee approximation (see eq. (12)), $\Theta\left(r_{12}-d\right)$ is the step function which describes the steric cut-off and the second term is the dipole-dipole interaction potential for bent-core molecules. Let us first consider the contribution from the dipole-dipole interaction to the second term in eq. (37). It should be noted that the dipole-dipole interaction is long range and one should be careful in averaging it over all positions and orientations of the polar molecules. As shown in our previous paper [39], the corresponding averaging in the polar nematic phase results in the following expression:

$$
\begin{aligned}
& \rho \int \Theta\left(r_{12}-d\right) U_{d d}\left(\mathbf{b}_{1}, \mathbf{b}_{2}, \mathbf{r}_{12}\right) f_{0}\left(\left(\mathbf{a}_{2} \cdot \mathbf{n}\right)^{2},\left(\mathbf{b}_{2} \cdot \mathbf{n}\right)^{2}\right) \\
& \times h\left(\mathbf{x}_{2}\right) \mathrm{d} \mathbf{x}_{2} \mathrm{~d} \mathbf{r}_{12}= \\
& -\frac{4 \pi}{3} \mu_{\perp}\left(\mathbf{b}_{1} \cdot \mathbf{P}\right)-\mu_{\perp}\left(\mathbf{b}_{1} \cdot \mathbf{E}\right)
\end{aligned}
$$

where $\mathbf{E}$ is the average electric field in the medium and the polarization $\mathbf{P}=\left\langle\rho \mu_{\perp} \mathbf{b}\right\rangle$. The contribution of the dipoledipole interaction to the first term in eq. (35) vanishes because the dipolar potential is quadratic in $\mathbf{u}_{12}$ while the factor $\left(\mathbf{r}_{12} \cdot \nabla\right)$ is odd in $\mathbf{u}_{12}$ and therefore the contribution vanishes after integration over $\mathbf{u}_{12}$.

In the first term in eq. (35) the integrand depends both on the intermolecular vector $\mathbf{r}_{12}$ and on the vector $\mathbf{r}_{i j}(=$ $\mathbf{r}_{\mu \nu}$ ). However, in this case one cannot approximately set $\mathbf{r}_{12}=\mathbf{r}_{i j}$ because as a result of this all polar terms, which contribute to flexoelectricity, will disappear. The vectors $\mathbf{r}_{12}$ and $\mathbf{r}_{i j}$ can be written in the form $\mathbf{r}_{12}=r_{12} \mathbf{u}_{12}$ and $\mathbf{r}_{i j}=r_{i j} \mathbf{u}_{i j}=\sigma_{i j} r_{i j}^{\prime} \mathbf{u}_{i j}$, where the difference between the unit vectors $\mathbf{u}_{i j}$ and $\mathbf{u}_{12}$ can be neglected because the integration is performed over the whole unit sphere. At the same time the difference between the modulus $r_{12}$ and $r_{i j}$ should be taken into consideration using the relationship $\mathbf{r}_{12}=\mathbf{r}_{i j}+L\left(\mathbf{a}_{i}-\mathbf{a}_{j}\right) / 2$ The modulus $r_{12}$ can be exressed as

$$
\begin{aligned}
r_{12}= & {\left[r_{i j}^{2}+\frac{L^{2}}{2}\left(1-\left(\mathbf{a}_{i} \cdot \mathbf{a}_{j}\right)\right)+L r_{i j}\left(\left(\mathbf{a}_{i} \cdot \mathbf{u}_{i j}\right)\right.\right.} \\
& \left.\left.-\left(\mathbf{a}_{j} \cdot \mathbf{u}_{i j}\right)\right)\right]^{1 / 2}
\end{aligned}
$$

and the differential $\mathrm{d} r_{12}$ can be written in the form:

$$
\begin{aligned}
& \mathrm{d} r_{12}=\frac{\mathrm{d} r_{12}}{\mathrm{~d} r_{i j}} \mathrm{~d} r_{i j}= \\
& \frac{r_{i j}+L\left(\left(\mathbf{a}_{i} \cdot \mathbf{u}_{i j}\right)-\left(\mathbf{a}_{j} \cdot \mathbf{u}_{i j}\right)\right)}{\left[r_{i j}^{2}+\frac{L^{2}}{2}\left(1-\left(\mathbf{a}_{i} \cdot \mathbf{a}_{j}\right)\right)+L r_{i j}\left(\left(\mathbf{a}_{i} \cdot \mathbf{u}_{i j}\right)-\left(\mathbf{a}_{j} \cdot \mathbf{u}_{i j}\right)\right)\right]^{1 / 2}} \\
& \times \mathrm{d} r_{i j} .
\end{aligned}
$$

Finally one obtains the following expression for $r_{12}^{2} \mathrm{~d} r_{12}$ which enters the integrals in (35):

$$
\begin{aligned}
r_{12}^{2} \mathrm{~d} r_{12}= & {\left[r_{i j}+L\left(\left(\mathbf{a}_{i} \cdot \mathbf{u}_{i j}\right)-\left(\mathbf{a}_{j} \cdot \mathbf{u}_{i j}\right)\right)\right] } \\
& \times\left[r_{i j}^{2}+\frac{L^{2}}{2}\left(1-\left(\mathbf{a}_{i} \cdot \mathbf{a}_{j}\right)\right)+L r_{i j}\left(\left(\mathbf{a}_{i} \cdot \mathbf{u}_{i j}\right)\right.\right. \\
& \left.\left.-\left(\mathbf{a}_{j} \cdot \mathbf{u}_{i j}\right)\right)\right]^{1 / 2} \mathrm{~d} r_{i j}
\end{aligned}
$$

Expanding eq. (41) in power of $L / r_{i j}$ one obtains

$$
\begin{aligned}
r_{12}^{2} \mathrm{~d} r_{12} \approx & r_{i j}^{2}\left[1+\frac{3}{2} \frac{L}{r_{i j}}\left(\left(\mathbf{a}_{i} \cdot \mathbf{u}_{i j}\right)-\left(\mathbf{a}_{j} \cdot \mathbf{u}_{i j}\right)\right)\right. \\
& \left.+\frac{L^{2}}{4 r_{i j}^{2}}\left(1-\left(\mathbf{a}_{i} \cdot \mathbf{a}_{j}\right)\right)\right] \mathrm{d} r_{i j}
\end{aligned}
$$

One notes that the middle term in brackets of eq. (42) is polar and odd in $\mathbf{u}_{i j}$ and thus they should contribute to the polar correction $h_{a}$ of the orientational distribution function.

Substituting eq. (42) and the equation $\mathbf{r}_{12}=\mathbf{r}_{i j}+$ $L\left(\mathbf{a}_{i}-\mathbf{a}_{j}\right) / 2$ into the first term in eq. (35), neglecting cubic terms in $\mathbf{a}_{i}$ and $\mathbf{a}_{j}$ and taking into account that only terms, which are odd in $\mathbf{u}_{i j}$, contribute to the integral, one obtains the following expression:

$$
\begin{aligned}
& -\frac{3}{2} \rho \lambda_{2} \sum_{i j} \int \sigma_{i j}^{3} L\left(\left(\mathbf{a}_{i} \cdot \mathbf{u}_{i j}\right)-\left(\mathbf{a}_{j} \cdot \mathbf{u}_{i j}\right)\right)\left(\mathbf{u}_{i j} \cdot \nabla\right) \\
& \times f_{0}\left(\left(\mathbf{a}_{2} \cdot \mathbf{n}\right)^{2},\left(\mathbf{b}_{2} \cdot \mathbf{n}\right)^{2}\right) \mathrm{d} \mathbf{a}_{2} \mathrm{~d} \mathbf{b}_{2} \mathrm{~d} \mathbf{u}_{i j},
\end{aligned}
$$

where

$$
\lambda_{2}=\int_{1}^{\infty} C_{2}\left(r_{i j}\right) r_{i j}^{2} \mathrm{~d} r_{i j} .
$$

Similar to sect. 3, the lowest-order term in the expansion of $\sigma_{i j}^{3}$ which contributes to the integral is $(L-d)^{2}(d / 3) P_{2}\left(\mathbf{a}_{\nu}\right.$. $\left.\mathbf{u}_{\nu \mu}\right) P_{2}\left(\mathbf{a}_{\mu} \cdot \mathbf{u}_{\nu \mu}\right)$ and thus eq. (43) can approximately be expressed as

$$
\begin{aligned}
& -\frac{\rho}{2} L d(L-d)^{2} \lambda_{2} \sum_{i j} \int P_{2}\left(\mathbf{a}_{\nu} \cdot \mathbf{u}_{\nu \mu}\right) P_{2}\left(\mathbf{a}_{\mu} \cdot \mathbf{u}_{\nu \mu}\right) \\
& \times\left(\left(\mathbf{a}_{i} \cdot \mathbf{u}_{i j}\right)-\left(\mathbf{a}_{j} \cdot \mathbf{u}_{i j}\right)\right)\left(\mathbf{u}_{i j} \cdot \nabla\right) \\
& \times f_{0}\left(\left(\mathbf{a}_{2} \cdot \mathbf{n}\right)^{2},\left(\mathbf{b}_{2} \cdot \mathbf{n}\right)^{2}\right) \mathrm{d} \mathbf{a}_{2} \mathrm{~d} \mathbf{b}_{2} \mathrm{~d} \mathbf{u}_{i j} .
\end{aligned}
$$


Finally, we consider the contribution of the direct correlation function between different molecular arms to the second term in the general eq. (35). One notes that the function $h\left(\mathbf{b}_{2}, \mathbf{a}_{2}\right)$ under the integral is polar in $\mathbf{b}_{2}$ and, in contrast to the first term in the same equation, there is no factor linear in $\mathbf{u}_{i j}$. As a result, in eq. (35) only terms which are odd in $\mathbf{b}_{2}$ and even in $\mathbf{u}_{i j}$ make a non-zero contribution. Substituting eq. (42) and the first term in eq. (37) into the second term in eq. (35) one obtains the following contribution:

$$
\begin{aligned}
& -\rho \lambda_{3} \sum_{i j} \int \sigma_{i j} \frac{L^{2}}{4}\left(\mathbf{a}_{i} \cdot \mathbf{a}_{j}\right) f_{0}\left(\left(\mathbf{a}_{2} \cdot \mathbf{n}\right)^{2},\left(\mathbf{b}_{2} \cdot \mathbf{n}\right)^{2}\right) \\
& \times h\left(\mathbf{b}_{2}, \mathbf{a}_{2}\right) \mathrm{d} \mathbf{a}_{2} \mathrm{~d} \mathbf{b}_{2} \mathrm{~d} \mathbf{u}_{i j},
\end{aligned}
$$

where

$$
\lambda_{3}=\int_{1}^{\infty} C_{2}\left(r_{i j}\right) \mathrm{d} r_{i j}
$$

and where $\sigma_{i j}$ is given by eq. (11).

One notes that the product $\left(\mathbf{a}_{i} \cdot \mathbf{a}_{j}\right)$ can be expressed as

$$
\begin{aligned}
\left(\mathbf{a}_{i} \cdot \mathbf{a}_{j}\right)= & \cos ^{2} \alpha\left(\mathbf{a}_{1} \cdot \mathbf{a}_{2}\right) \pm \cos \alpha \sin \alpha \\
& \times\left(\left(\mathbf{a}_{1} \cdot \mathbf{b}_{2}\right)+\left(\mathbf{a}_{2} \cdot \mathbf{b}_{1}\right)\right)+\sin ^{2} \alpha\left(\mathbf{b}_{1} \cdot \mathbf{b}_{2}\right) .
\end{aligned}
$$

In this expression only the last term, which is polar both in $\mathbf{b}_{1}$ and $\mathbf{b}_{2}$, makes a non-zero contribution. Other terms vanish after averaging over $\mathbf{b}_{2}$ or $\mathbf{b}_{1}$ in the final expression for the spontaneous polarization.

Combining eqs. (38), (43), (46) then polar correction to the orientational distribution function of the polar bentcore nematic can be expressed as:

$$
\begin{aligned}
h_{a}\left(\mathbf{b}_{1}, \mathbf{a}_{1}\right)= & \frac{4 \pi}{3 k_{B} T} \mu_{\perp}\left(\mathbf{b}_{1} \cdot \mathbf{P}\right)+\frac{1}{k_{B} T} \mu_{\perp}\left(\mathbf{b}_{1} \cdot \mathbf{E}\right) \\
& -\frac{\rho}{12} L^{2}(L-d) \lambda_{3} \int\left[\left(\mathbf{a}_{1} \cdot \mathbf{u}\right)^{2}+\left(\mathbf{a}_{2} \cdot \mathbf{u}\right)^{2}\right] \\
& \times \sin ^{2} \alpha\left(\mathbf{b}_{1} \cdot \mathbf{b}_{2}\right) f_{0}\left(\left(\mathbf{a}_{2} \cdot \mathbf{n}\right)^{2},\left(\mathbf{b}_{2} \cdot \mathbf{n}\right)^{2}\right) \\
& \times h\left(\mathbf{b}_{2}, \mathbf{a}_{2}\right) \mathrm{d} \mathbf{a}_{2} \mathrm{~d} \mathbf{b}_{2} \mathrm{~d} \mathbf{u} \\
& -\frac{1}{2} \rho L d(L-d)^{2} \lambda_{2} \int P_{2}\left(\mathbf{a}_{2} \cdot \mathbf{u}\right) P_{2}\left(\mathbf{a}_{1} \cdot \mathbf{u}\right) \\
& \times\left(\left(\mathbf{a}_{1} \cdot \mathbf{u}\right)-\left(\mathbf{a}_{2} \cdot \mathbf{u}\right)\right)(\mathbf{u} \cdot \nabla) \\
& \times f_{0}\left(\left(\mathbf{a}_{2} \cdot \mathbf{n}\right)^{2},\left(\mathbf{b}_{2} \cdot \mathbf{n}\right)^{2}\right) \mathrm{d} \mathbf{a}_{2} \mathrm{~d} \mathbf{b}_{2} \mathrm{~d} \mathbf{u} .
\end{aligned}
$$

Now the polarization $\mathbf{P}$ can be expressed as:

$$
\begin{aligned}
\mathbf{P}= & \rho \int \mu_{\perp} \mathbf{b} f_{0}\left((\mathbf{a} \cdot \mathbf{n})^{2},(\mathbf{b} \cdot \mathbf{n})^{2}\right) h(\mathbf{b}, \mathbf{a}) \mathrm{d} \mathbf{a} \mathrm{d} \mathbf{b} \\
= & \frac{\pi}{3} \frac{\rho \mu_{\perp}^{2}}{2 k_{B} T} \mathbf{P}+\frac{\rho \mu_{\perp}^{2}}{2 k_{B} T} \mathbf{E} \\
& -\frac{1}{2} \rho^{2} \mu_{\perp} \lambda_{2} \sin \alpha L d(L-d) \tilde{S}^{2} \int \frac{1}{2}\left[\frac{1}{3}(2+S) \mathbf{u}\right. \\
& -S \mathbf{n}(\mathbf{n} \cdot \mathbf{u})] P_{2}(\mathbf{n} \cdot \mathbf{u})(\mathbf{u} \cdot \nabla) P_{2}(\mathbf{n} \cdot \mathbf{u}) \mathrm{d} \mathbf{u} \\
& +\frac{1}{24} \rho \lambda_{3} \sin ^{2} \alpha L^{2}(L-d) \tilde{S} \int\left[\frac{1}{3}(2+S) \mathbf{P}\right. \\
& -S \mathbf{n}(\mathbf{n} \cdot \mathbf{P})](\mathbf{n} \cdot \mathbf{u})^{2} \mathrm{~d} \mathbf{u},
\end{aligned}
$$

where $(\mathbf{u} \cdot \nabla) P_{2}(\mathbf{n} \cdot \mathbf{u})=3(\mathbf{n} \cdot \mathbf{u})(\mathbf{u} \cdot \nabla)(\mathbf{n} \cdot \mathbf{u})$. Here we have decoupled the averages over short and long molecular axes (thus neglecting the corresponding third rank polar order parameters). In this case $\left\langle b_{\alpha} b_{\beta}\right\rangle=\left(\delta_{\alpha \beta}-\left\langle a_{\alpha} a_{\beta}\right\rangle\right) / 2=$ $\left[(1 / 3)(2+S) \delta_{\alpha \beta}-S n_{\alpha} n_{\beta}\right]$ if the order parameter $D=0$.

Let us assume for simplicity that the director varies slowly in the $(x, z)$ plane and that the local director $\mathbf{n} \| \mathbf{z}$. Then in the case of pure splay the flexoelectric polarization $\mathbf{P}\|\mathbf{n}\| \mathbf{z}$ and the only non-zero component of splay is $\nabla_{x} n_{x}$. In this case $P=P_{z}=\tilde{e}_{11} \nabla_{x} n_{x}$, where $\tilde{e}_{11}$ is the effective splay flexocoefficient. Substituting these components into eq. (49) and integrating over $\mathbf{u}$ one obtains the following expression for $\tilde{e}_{11}$ :

$$
\tilde{e}_{11}=e_{11}\left(1-\frac{1}{3}\left(4 \pi \chi_{0}-2(1+2 S) A_{0} \sin ^{2}(\alpha)\right)\right)^{-1}
$$

where

$$
A_{0}=\frac{\rho \lambda_{3}}{108} L^{2}(L-d)=\frac{\rho^{*} \lambda_{3}}{108} \frac{L(L-d)}{d^{2}} \equiv \frac{\epsilon}{108} \frac{L(L-d)}{d^{2}},
$$

and

$$
\chi_{0}=\frac{\rho \mu_{\perp}^{2}}{k_{B} T}=\frac{\rho \mu_{\perp}^{2}}{t J} \equiv \frac{\psi_{0}}{t} .
$$

Here $e_{11}$ is the so called bare flexoelectric coefficient, which enters the equations of the phenomenological theory and which is obtained by neglecting the last two terms in the denominator in eq. (50):

$$
e_{11}=-\frac{8 \pi}{315} \nu \chi_{0}^{-1} \sin (\alpha) \tilde{S}^{2}(1-S)
$$

where

$$
\nu=\frac{2}{3} \rho^{2} \lambda_{2} \mu_{\perp} d L(L-d)^{2} .
$$

In the case of pure bend $\mathbf{P} \| \mathbf{x} \perp \mathbf{n}$ and the only non-zero component of bend is $\nabla_{z} n_{x}$. Here $P=P_{x}=\tilde{e}_{33} \nabla_{z} n_{x}$, where $\tilde{e}_{33}$ is the effective bend flexocoefficient. Substituting these components into eq. (49) one obtains the following expression for $\tilde{e}_{33}$ :

$$
\tilde{e}_{33}=e_{33}\left(1-\frac{1}{3}\left(4 \pi \chi_{0}-(2+S) A_{0} \sin ^{2}(\alpha)\right)\right)^{-1},
$$

where $e_{33}$ is the undressed bend flexocoefficient:

$$
e_{33}=-\frac{8 \pi}{315} \nu \chi_{0}^{-1} \sin (\alpha) \tilde{S}^{2}(2+S) .
$$

The effective and undressed flexocoefficients are presented in the reduced form divided by the factor $\frac{8 \pi}{315} \nu$, which makes the bare coefficients depending only on parameter $\alpha$ and dimensionless temperature $t$. Additionally for the effective flexocoefficients we also introduce the parameters $\rho^{*}=\rho d^{2} L, \epsilon$ and $\psi_{0}$ which are defined in the eqs. (51) and (52) respectively. The results for bare flexocoefficients are presented in fig. 3 , where the red line is the reference of rod-like particle with zero value. The absolute values of $e_{33}$ are approximately an order of magnitude larger than $e_{11}$, and as expected the latter goes to 
a)

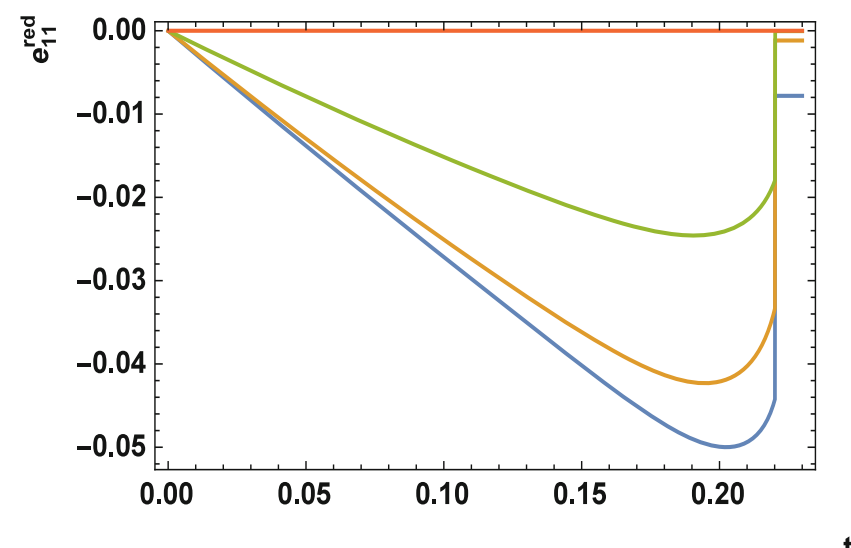

b)

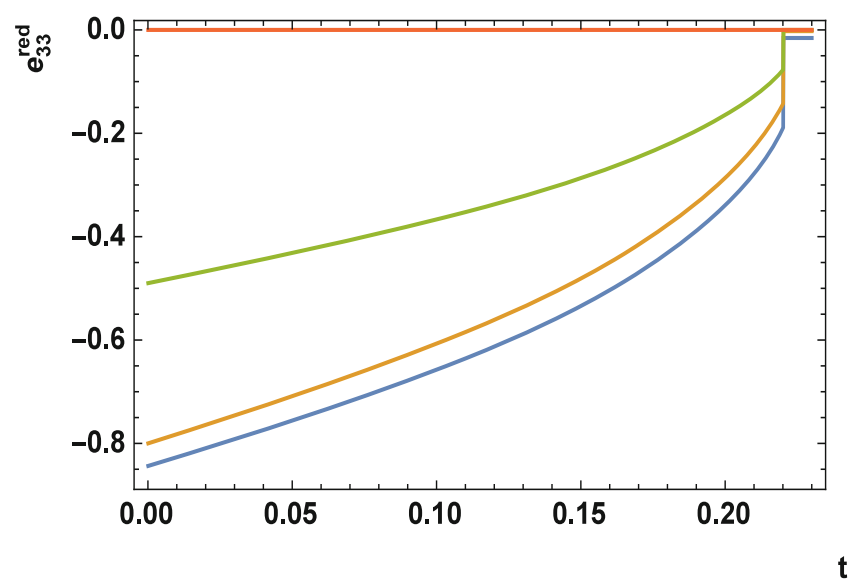

Fig. 3. (Color online) Reduced splay (a) and bend (b) flexoelectric coefficients $e_{i i}^{\text {red }}=\frac{315 \chi_{0}}{8 \pi \nu} e_{i i}$, where $e_{i i}$ are given by eq. (53) and eq. (56), for four opening angles from the very top: $180^{\circ}$ (red), $160^{\circ}$ (green), $140^{\circ}$ (orange) and $120^{\circ}$ (blue), which correspond to parameter $\alpha$ equal to $0, \frac{\pi}{18}, \frac{\pi}{9}$ and $\frac{\pi}{6}$, respectively.

zero when temperature reaches zero. The dressed flexocoefficients are also obtained as the same reduced variables for various values of $\psi_{0}$ and presented for $\alpha=\frac{\pi}{6}, L=2.5 d$ and $\epsilon=75$ in fig. 4 and fig. 5 . In these figures flexoelectric coefficients tend to diverge at the temperature within the nematic phase range, which is caused by a transition to the possibly virtual polar state and indeed it is governed by a larger value of $\psi_{0}$, that is by stronger dipole-dipole interactions eq. (52). Here we present outcomes for just one opening angle, as the effect is the same for other values of parameter $\alpha$. The role of $\epsilon$ is also secondary, because it affects mainly how fast flexocoefficients diverge, that is the steepness of curves in fig. 4 and fig. 5 . In the next section we present how this effect influences the elastic properties in this model of bent-core molecules.

\section{Effective elastic constants}

Equation (49) for the polarization $\mathbf{P}$ can also be used to obtain the longitudinal and the transverse components of

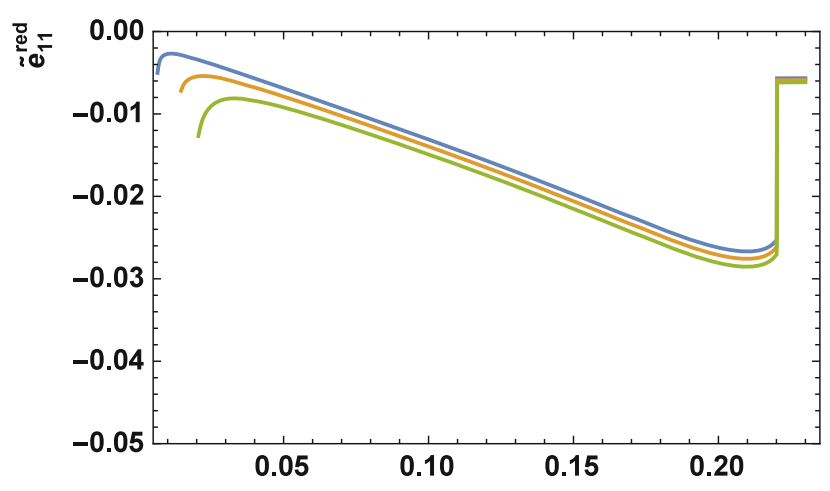

Fig. 4. (Color online) Effective reduced splay flexoelectric coefficients $\tilde{e}_{11}^{\text {red }}=\frac{315 \chi_{0}}{8 \pi \nu} \tilde{e}_{11}$, where $\tilde{e}_{11}$ are given by eq. (50) for three values of $\psi_{0}$ from the very top: 0.003 (blue), 0.006 (orange) and 0.009 (green). The other parameters of the model are $\epsilon=75, L=2.5 d$ and $\alpha=\frac{\pi}{6}$.

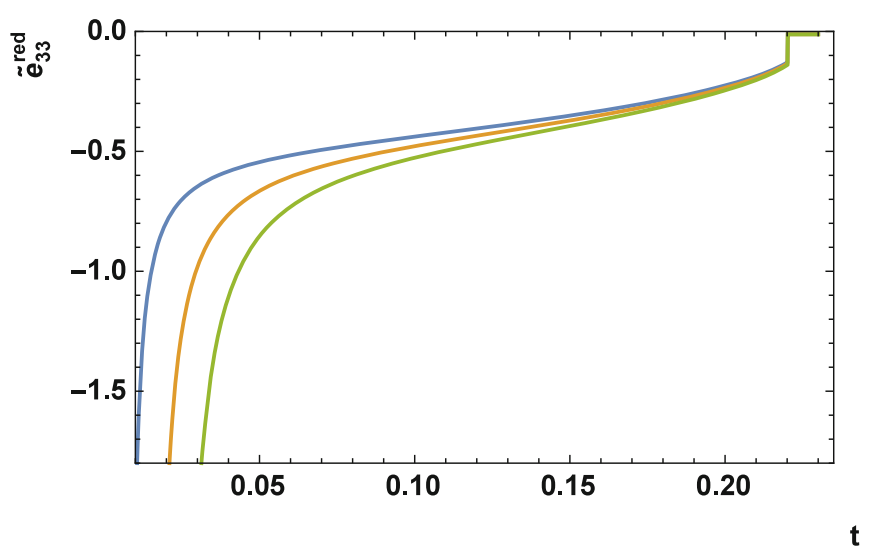

Fig. 5. (Color online) Effective reduced bend flexoelectric coefficients $\tilde{e}_{33}^{\text {red }}=\frac{315 \chi_{0}}{8 \pi \nu} \tilde{e}_{33}$, where $\tilde{e}_{33}$ are given by eq. (55) for three values of $\psi_{0}$ from the very top: 0.003 (blue), 0.006 (orange) and 0.009 (green). The other parameters of the model are $\epsilon=75, L=2.5 d$ and $\alpha=\frac{\pi}{6}$.

the dielectric susceptibility:

$$
\chi_{\|}=\chi_{0}\left(1-\frac{1}{3}\left(4 \pi \chi_{0}-2(1+2 S) A_{0} \sin ^{2}(\alpha)\right)\right)^{-1},
$$

and

$$
\chi_{\perp}=\chi_{0}\left(1-\frac{1}{3}\left(4 \pi \chi_{0}-(2+S) A_{0} \sin ^{2}(\alpha)\right)\right)^{-1},
$$

Now the effective elastic constants are given by the following equations:

$$
\tilde{K}_{11}=K_{11}-\frac{1}{2} \chi_{\|} e_{11}^{2} ; \quad \tilde{K}_{33}=K_{33}-\frac{1}{2} \chi_{\perp} e_{33}^{2},
$$

where the bare elastic constants are given by eqs. (53) and (56), and $\chi_{0}$ is expressed by eq. (52).

The so-called bare elastic constants, given by eqs. (27) and (28), are presented in fig. 2. The bare constants are not renormalized by the polar intermolecular interactions 


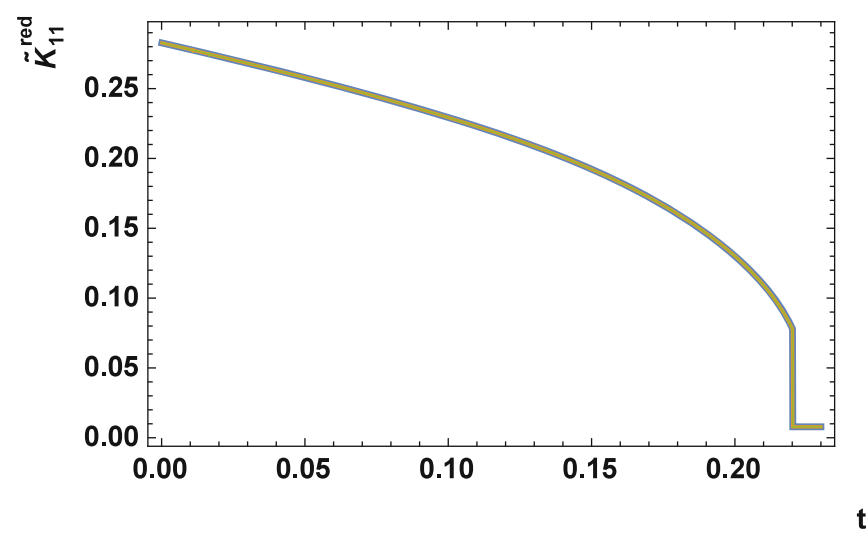

Fig. 6. (Color online) Effective reduced splay elastic constant $\tilde{K}_{11}^{\text {red }}=\frac{d}{-\lambda t J\left(\rho^{*}\right)^{2}} \tilde{K}_{11}$, where $\tilde{K}_{11}$ are given by eq. (59) for three values of $\psi_{0}$ : 0.003 (blue), 0.006 (orange) and 0.009 (green), which coincide and are indistinguishable from the bare $K_{11}$ shown in fig. 2(a). The other parameters of the model are $\epsilon=$ $75, L=2.5 d$ and $\alpha=\frac{\pi}{6}$.

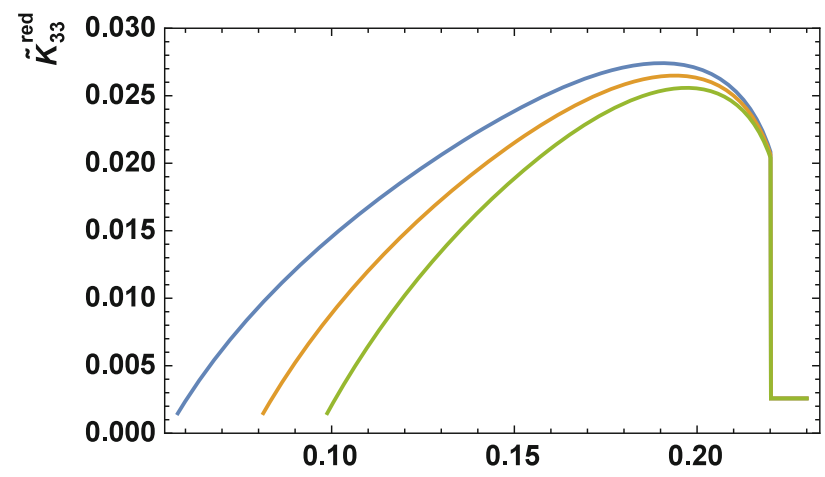

Fig. 7. (Color online) Effective reduced bend elastic constant $\tilde{K}_{33}^{\text {red }}=\frac{d}{-\lambda t J\left(\rho^{*}\right)^{2}} \tilde{K}_{33}$, where $\tilde{K}_{33}$ are given by eq. (59) for three values of $\psi_{0}$ from the very top: 0.003 (blue), 0.006 (orange) and 0.009 (green). The other parameters of the model are $\epsilon=75$, $L=2.5 d$ and $\alpha=\frac{\pi}{6}$.

and as a result they possess a standard temperature dependence, i.e. grow monotonically with the decreasing temperature. This growth is mainly determined by the increase of the nematic order parameter $S$. The bare flexoelectric coefficients presented in fig. 3 also are not characterized by any unexpected temperature variation. The bend coefficient is increasing with the decreasing temperature, while the splay coefficient decreases. This decrease is determined by the factor $(S-1)$ in eq. (53) which vanishes when $S=1$. This is related to the fact that in our molecular model the bent-core molecule possesses only the transverse dipole. In the case of perfect orientational order all transverse dipoles are perpendicular to the director and hence the splay flexoelectric polarization must vanish.

In contrast, the effective flexocoefficients behave in a different way. One can readily see from fig. 4 that the values of the splay flexocoefficient practically do not differ

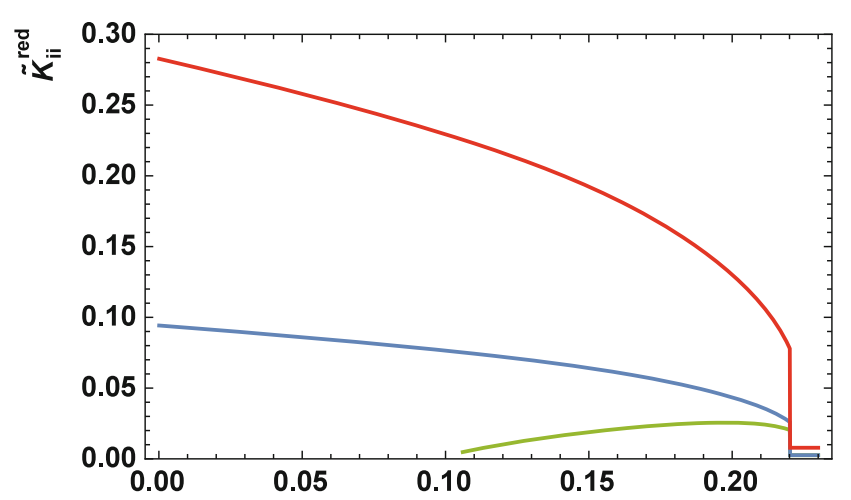

$\mathbf{t}$

Fig. 8. (Color online) Effective reduced elastic constant $\tilde{K}_{i i}^{\text {red }}=\frac{d}{-\lambda t J\left(\rho^{*}\right)^{2}} \tilde{K}_{i i}$ obtained for $\epsilon=75, L=2.5 d, \alpha=\frac{\pi}{6}$ and $\psi_{0}=0.009$, where the red curve is for splay elastic constants, blue and green lines for, respectively, bare and effective bend elastic constants.

from those of the bare one, while the bend coefficient is much bigger than the bare one and formally diverges at some low temperature. This divergence is determined by the factor $\left(1-\frac{1}{3}\left(4 \pi \chi_{0}-(2+S) A_{0} \sin ^{2}(\alpha)\right)\right)^{-1}$ in eq. (55) which is determined by polar intermolecular interactions. The divergence point can be interpreted as a temperature of a transition into the virtual ferroelectric phase. In real systems such a transition never occurs because the material crystallizes or undergoes a transition into the smectic phase. Thus the anomalously large values of the bend flexocoefficient are determined by a "pretransitional effect".

As a result the absolute value of the negative flexoelectric contribution to the effective bend elastic constant appears to be rather large at sufficiently low temperatures. The bend elastic constant is very small everywhere in the nematic phase and approaches zero at some temperature as shown in fig. 7. Here the effective elastic constants are plotted in reduced units, i.e. $\tilde{K}_{i i}^{\text {red }}=\frac{d}{-\lambda t J\left(\rho^{*}\right)^{2}} \tilde{K}_{i i}$ provided that $\lambda_{2}=-\lambda$. Different curves correspond to different values of the parameter $\psi_{0}$, whole the remaining parameters of the model are: $\alpha=\frac{\pi}{6}, L=2.5 d$ and $\epsilon=75$.

One notes that due to the anisotropy of the nematic phase the factors $\left(1-\frac{1}{3}\left(4 \pi \chi_{0}-(2+S) A_{0} \sin ^{2}(\alpha)\right)\right)^{-1}$ and $\left(1-\frac{1}{3}\left(4 \pi \chi_{0}-2(1+2 S) A_{0} \sin ^{2}(\alpha)\right)\right)^{-1}$ in the equations for the bend and splay flexocoefficients, respectively may behave in a qualitatively different way. In particular, for small values of $\psi_{0}$ the second term in the denominator of the latter factor is negative and therefore the factor does not diverge. In this case the splay flexocoefficient remains small and the effective splay elastic constant is approximately equal to the bare elastic constant as can be seen in fig. 6 and fig. 2(a). For larger values of the parameter $\psi_{0}$ the bend elastic constant is smaller. Taking into account that $\psi_{0}$, given by eq. (52), is proportional to the square of the molecular electric dipole, one concludes that the abnormally small values of the bend elastic constant may be promoted by increasing the electric dipole of a bent-core molecule. 
In fig. 8 both splay and bend effective elastic constants are presented in the same units. One can readily see that the splay constant behaves in a standard way, i.e. it increases monotonically with the decreasing temperature, while the bend constant is everywhere very small and approaches zero at some temperature. This behavior is qualitatively very similar to the one observed for instance in $[3,8,15]$.

\section{Discussion}

In this paper we have developed a molecular-statistical theory of both flexoelectricity and the elastic constants of the nematic composed of bent-core molecules using the same molecular model and the Parsons-Lee approximation for intermolecular correlations. It has been shown that polar intermolecular interactions, including the electrostatic dipole-dipole interactions, make a significant contribution to the flexoelectric coefficients. If this contribution is not taken into consideration, however, the flexocoefficients appear to be moderately large and possess standard temperature variation. On the other hand, sufficiently strong polar interactions between bent-core molecules result in a dramatic increase of the bend flexocoefficient which may even diverge at a certain low temperature which corresponds to a transition into the virtual ferroelectric phase. One notes, however, that this phase is never observed because the corresponding transition temperature is far below the nematic range. At the same time the splay flexocoefficient is only weakly renormalized.

As a result the flexoelectric correction to the splay elastic constant is very small and thus this constant is practically not affected by polar interactions between bent-core molecules. In contrast, the flexoelectric correction to the bend elastic constant may be sufficiently large which results in a very low values of the bend constant throughout the nematic range. The negative flexoelectric correction may even drive the bend elastic constant at some temperature which corresponds to an elastic instability leading to a transition into the twist-bend phase $[12,16]$. These results correspond to the existing experimental data.

It should be noted that in the general framework of the Selinger model [16], which has been investigated here from the molecular point if view, the abnormally large values of the flexocoefficients are related to the "pretransitional" increase of the mean-field dielectric susceptibility which is not generally observed in bent-core nematics [40]. On the other hand, molecular theory of the dielectric constant of nematic LCs should be more complicated because dipole-dipole correlations are expected to give a significant contribution. However, in some bent-core nematics the dielectric constant possesses a very weak temperature variation in the vicinity of the transition into the TB phase. This contradiction may be resolved if one takes into consideration that the present theory in its general form is valid even if the molecules do not possess electric dipoles at all. Bent-core molecules generally possess transverse steric dipoles which will promote some polar intermolecular interactions. These interactions in turn, determine the polar correction to the orientational distribution function which contributes to the bend elastic constant leading to its reduction. This may explain why "negative" values of the elastic constants have been obtained by Ferrarini et al. $[22]$.

We are grateful to A. Ferrarini and G. R. Luckhurst for interesting discussions. This work was partly supported by the Grant No. DEC-2013/11/B/ST3/04247 of the National Science Centre in Poland.

Open Access This is an open access article distributed under the terms of the Creative Commons Attribution License (http://creativecommons.org/licenses/by/4.0), which permits unrestricted use, distribution, and reproduction in any medium, provided the original work is properly cited.

\section{References}

1. V.P. Panov, M. Nagaraj, J.K. Vij, Yu.P. Panarin, A. Kohlmeier, M.G. Tamba, R.A. Lewis, G.H. Mehl, Phys. Rev. Lett. 105, 167801 (2010).

2. M. Cestari, S. Diez-Berart, D.A. Dunmur, A. Ferrarini, M.R. de la Fuente, D.J.B. Jackson, D.O. Lopez, G.R. Luckhurst, M.A. Perez-Jubindo, R.M. Richardson, J. Salud, B.A. Timimi, H. Zimmermann, Phys. Rev. E 84, 031704 (2011).

3. V. Borshch, Y.-K. Kim, J. Xiang, M. Gao, A. Jákli, V.P. Panov, J.K. Vij, C.T. Imrie, M.G. Tamba, G.H. Mehl, O.D. Lavrentovich, Nat. Commun. 4, 2635 (2013).

4. V. Görtz, C. Southern, N.W. Roberts, H.F. Gleeson, J.W. Goodby, Soft Matter 5, 463 (2009).

5. D. Chen, M. Nakata, R. Shao, M.R. Tuchband, M. Shuai, U. Baumeister, W. Weissflog, D.M. Walba, M.A. Glaser, J.E. Maclennan, N.A. Clark, Phys. Rev. E 89, 022506 (2014).

6. Y. Wang, G. Singh, D.M. Agra-Kooijman, M. Gao, H.K. Bisoyi, C. Xue, M.R. Fisch, S. Kumar, Q. Li, CrystEngComm 17, 2778 (2015).

7. D. Chen, J.H. Porada, J.B. Hooper, A. Klittnick, Y. Shen, M.R. Tuchband, E. Korblova, D. Bedrov, D.M. Walba, M.A. Glaser, J.E. Maclennan, N.A. Clark, Proc. Natl. Acad. Sci. U.S.A. 110, 15931 (2013).

8. K. Adlem, M. Čopič, G.R. Luckhurst, A. Mertelj, O. Parri, R.M. Richardson, B.D. Snow, B.A. Timimi, R.P. Tuffin, D. Wilkes, Phys. Rev. E 88, 022503 (2013).

9. B. Robles-Hernández, N. Sebastián, M. Rosario de la Fuente, D.O. López, S. Diez-Berart, J. Salud, M. Blanca Ros, D.A. Dunmur, G.R. Luckhurst, B.A. Timimi, Phys. Rev. E 92, 062505 (2015).

10. V.L. Lorman, B. Mettout, Phys. Rev. Lett. 82, 940 (1999).

11. T.C. Lubensky, L. Radzihovsky, Phys. Rev. E 66, 031704 (2002).

12. I. Dozov, Europhys. Lett. 56, 247 (2001).

13. R. Memmer, Liq. Cryst. 29, 483 (2002).

14. C. Greco, A. Ferrarini, Phys. Rev. Lett. 115, 147801 (2015).

15. C.-J. Yun, M.R. Vengatesan, J.K. Vij, J.-K. Song, Appl. Phys. Lett. 106, 173102 (2015).

16. S.M. Shamid, S. Dhakal, J.V. Selinger, Phys. Rev. E 87, 052503 (2013). 
17. J. Harden, B. Mbanga, N. Éber, K. Fodor-Csorba, S. Sprunt, J.T. Gleeson, A. Jákli, Phys. Rev. Lett. 97, 157802 (2006).

18. A. Jákli, J. Harden, N. Éber, Flexoelectricity of bent-core molecules, in Flexoelectricity in liquid crystals. Theory, Experiment and Applications, edited by Agnes Buka, Nandor Éber (Emperial College Press, 2013).

19. N. Vaupotič, S. Curk, M.A. Osipov, M. Cepič, H. Takezoe, Ewa Gorecka, Phys. Rev. E 93, 022704 (2016).

20. N. Vaupotič, M. Čepič, M.A. Osipov, E. Gorecka, Phys. Rev. E 89, 030501(R) (2014).

21. L. Longa, G. Pająk, Phys. Rev. E 93, 040701(R) (2016).

22. C. Greco, G.R. Luckhurst, A. Ferrarini, Soft Matter 10 9318 (2014).

23. P.I.C. Teixeira, A.J. Masters, B.M. Mulder, Mol. Cryst. Liq. Cryst. 323, 167 (1997)

24. A.G. Vanakaras, D.J. Photinos, Soft Matter 12, 2208 (2016).

25. R.B. Meyer, Phys. Rev. Lett. 22, 918 (1969).

26. M.A. Osipov, Molecular theory of flexoelectricity in nematic liquid crystals, in Flexoelectricity in liquid crystals. Theory, Experiment and Applications, edited by Agnes Buka, Nandor Éber (Emperial College Press, 2013).

27. Y. Singh, S. Singh, K. Rajesh, Phys. Rev. A 45, 974 (1991).
28. T.J. Sluckin, P. Shukla, J. Phys. A: Math. Gen. 16, 1539 (1983).

29. M.A. Osipov, Molecular Theories of Liquid Crystals, in Handbook of Liquid Crystals, Fundamentals of Liquid Crystals, edited by J.W. Goodby, P.J. Collins, T. Kato, C. Tschierske, H.F. Gleeson, P. Raynes, Vol. 1 (WileyVCH Verlag GmbH Co. KGaA, Weinheim, Germany, 2014) pp. $115-168$.

30. W.M. Gelbart, A. Ben-Shaul, J. Chem. Phys. 77, 916 (1982).

31. A.M. Somosa, P. Tarazona, Mol. Phys. 72, 911 (1991).

32. A. Poniewierski, J. Stecki, Mol. Phys. 38, 1931 (1979).

33. M.A. Osipov, S. Hess, Mol. Phys. 78, 1191 (1993).

34. A. Srivastava, S. Singh, J. Phys.: Condens. Matter 16, 7169 (2004).

35. A. Kapanowski, Phys. Rev. E 75, 031709 (2007).

36. J.D. Parsons, Phys. Rev. A 19, 1225 (1979).

37. S.D. Lee, J. Chem. Phys. 89, 7036 (1987).

38. B.W. van der Meer, G. Vertogen, in The Molecular Physics of Liquid Crystals, edited by G.R. Luckhurst, G.W. Gray (Academic Press, 1979).

39. M.A. Osipov, G. Pająk, Eur. Phys. J. E 37, 79 (2014).

40. S.P. Sreenilayam, Yu.P. Panarin, J.K. Vij, S.I. Torgova, A. Lehmann, C. Tschierske, Phys. Rev. E 92, 022502 (2015). 